\title{
Necessary and sufficient conditions for a class of functions and their reciprocals to be logarithmically completely monotonic
}

\author{
Yu-Pei Lv, Tian-Chuan Sun and Yu-Ming Chu*
}

\author{
* Correspondence: \\ chuyuming2005@yahoo.com.cn \\ Department of Mathematics, \\ Huzhou Teachers College, Huzhou \\ 313000, PR China
}

\begin{abstract}
We prove that the function $F_{\alpha, \beta}(x)=x^{\alpha} \Gamma^{\beta}(x) / \Gamma(\beta x)$ is strictly logarithmically completely monotonic on $(0, \infty)$ if and only if $(\alpha, \beta) \in\{(\alpha, \beta): \beta>0, \beta \geq 2 \alpha+1, \beta \geq$ $\alpha+1\} \backslash\{(\alpha, \beta): \alpha=0, \beta=1\}$ and that $\left[F_{\alpha, \beta}(x)\right]^{-1}$ is strictly logarithmically completely monotonic on $(0, \infty)$ if and only if $(\alpha, \beta) \in\{(\alpha, \beta): \beta>0, \beta \leq 2 \alpha+1, \beta \leq \alpha+1\}$ $\backslash\{(\alpha, \beta): \alpha=0, \beta=1\}$.

2010 Mathematics Subject Classification: 33B15; 26A48.

Keywords: completely monotonic, logarithmically completely monotonic, gamma function
\end{abstract}

\section{Introduction}

For real and positive values of $x$ the Euler gamma function $\Gamma$ and its logarithmic derivative $\psi$, the so-called digamma functions are defined by

$$
\begin{aligned}
& \Gamma(x)=\int_{0}^{\infty} t^{x-1} e^{-t} d t \\
& \psi(x)=\frac{\Gamma^{\prime}(x)}{\Gamma(x)}=-\gamma+\int_{0}^{\infty} \frac{e^{-t}-e^{-x t}}{1-e^{-t}} d t
\end{aligned}
$$

where $\gamma=0.5772 \ldots$ is the Euler's constant.

For extension of these functions to complex variable and for basic properties see [1] Over the last half century, many authors have established inequalities and monotonicity for these functions [2-22].

We know that a real-valued function $f: I \rightarrow \mathbb{R}$ is said to be completely monotonic on $I$ if $f$ has derivatives of all orders on $I$ and

$$
(-1)^{n} f^{(n)}(x) \geq 0
$$

for all $x \in I$ and $n \geq 0$. Moreover, $f$ is said to be strictly completely monotonic if inequalities (1.3) are strict.

We also know that a positive real-valued function $f: I \rightarrow(0, \infty)$ is said to be logarithmically completely monotonic on $I$ if $f$ has derivatives of all orders on $I$ and its logarithm $\log f$ satisfies

\section{空




$$
(-1)^{k}[\log f(x)]^{(k)} \geq 0
$$

for all $x \in I$ and $k \in \mathbb{N}$. Moreover, $f$ is said to be strictly logarithmically completely monotonic if inequalities (1.4) are strict.

Recently, the completely monotonic or logarithmically completely monotonic functions have been the subject of intensive research. In particular, many complete monotonicity and logarithmically complete monotonicity properties related to the gamma function, psi function, and polygamma function can be found in the literature [17,18,23-37]. In 1997, Merkle [38] proved that $F(x)=\frac{\Gamma(2 x)}{\Gamma^{2}(x)}$ is strictly log-concave on $(0, \infty)$. Later, Chen [39] showed that $[F(x)]^{-1}=\frac{\Gamma^{2}(x)}{\Gamma(2 x)}$ is strictly logarithmically completely monotonic on $(0, \infty)$. In [40], Li and Chen proved that $F_{\beta}(x)=\frac{\Gamma^{\beta}(x)}{\Gamma(\beta x)}$ is strictly logarithmically completely monotonic on $(0, \infty)$ for $\beta>1$, and that $\left[F_{\beta}(x)\right]^{-1}$ is strictly logarithmically completely monotonic on $(0, \infty)$ for $0<\beta<1$. The purpose of this article is to generalize $\mathrm{Li}$ and Chen's result. Our main result is as follows.

Theorem 1.1 Let $\alpha \in \mathbb{R}, \beta>0$ and $F_{\alpha, \beta}(x)=x^{\alpha} \Gamma^{\beta}(x) / \Gamma(\beta x)$, then

(1) $F_{\alpha, \beta}(x)$ is strictly logarithmically completely monotonic on $(0, \infty)$ if and only if $(\alpha, \beta) \in\{(\alpha, \beta): \beta>0, \beta \geq 2 \alpha+1, \beta \geq \alpha+1\} \backslash\{(\alpha, \beta): \alpha=0, \beta=1\} ;$

(2) $\left[F_{\alpha, \beta}(x)\right]^{-1}$ is strictly logarithmically completely monotonic on $(0, \infty)$ if and only if $(\alpha, \beta) \in\{(\alpha, \beta): \beta>0, \beta \leq 2 \alpha+1, \beta \leq \alpha+1\} \backslash\{(\alpha, \beta): \alpha=0, \beta=1\}$.

\section{Lemma}

In order to prove our Theorem 1.1, we need a lemma which we present in this section.

Lemma 2.1 Let $\alpha \in \mathbb{R}, \beta \in(0,1) \cup(1, \infty)$ and

$$
h(t)=-\alpha e^{-(\beta+1) t}+(\alpha+1) e^{-\beta t}+(\alpha-\beta) e^{-t}+\beta-\alpha-1 .
$$

Then the following statements are true:

(1) If $\beta \leq \alpha+1$ and $\beta \leq 2 \alpha+1$, then $h(t)<0$ for $t \in(0, \infty)$;

(2) If $\alpha+1<\beta<2 \alpha+1$, then there exists $\lambda_{1} \in(0, \infty)$ such that $h(t)<0$ for $t \in(0$,

$\left.\lambda_{1}\right)$ and $h(t)>0$ for $t \in\left(\lambda_{1}, \infty\right)$;

(3) If $\beta \geq \alpha+1$ and $\beta \geq 2 \alpha+1$, then $h(t)>0$ for $t \in(0, \infty)$;

(4) If $2 \alpha+1<\beta<\alpha+1$, then there exists $\lambda_{2} \in(0, \infty)$ such that $h(t)>0$ for $t \in(0$, $\left.\lambda_{2}\right)$ and $h(t)<0$ for $t \in\left(\lambda_{2}, \infty\right)$.

Proof Let $h_{1}(\mathrm{t})=e^{(\beta+1) t} h^{\prime}(\mathrm{t})$ and $h_{2}(t)=e^{-t} h_{1}^{\prime}(t)$. Then simple computations lead to

$$
\begin{aligned}
h(0) & =0, \\
h^{\prime}(t) & =\alpha(\beta+1) e^{-(\beta+1) t}-\beta(\alpha+1) e^{-\beta t}-(\alpha-\beta) e^{-t}, \\
h_{1}(0) & =h^{\prime}(0)=0, \\
h_{1}(t) & =\alpha(\beta+1)-\beta(\alpha+1) e^{t}-(\alpha-\beta) e^{\beta t}, \\
h^{\prime}{ }_{1}(t) & =-\beta(\alpha+1) e^{t}-\beta(\alpha-\beta) e^{\beta t},
\end{aligned}
$$




$$
\begin{aligned}
& h_{2}(0)=h_{1}^{\prime}(0)=\beta(\beta-2 \alpha-1) \\
& h_{2}(t)=-\beta(\alpha+1)-\beta(\alpha-\beta) e^{(\beta-1) t}
\end{aligned}
$$

and

$$
h_{2}^{\prime}(t)=\beta(\beta-1)(\beta-\alpha) e^{(\beta-1) t} .
$$

(1) If $\beta \leq \alpha+1$ and $\beta \leq 2 \alpha+1$, then we divide the proof into four cases.

Case 1 If $0<\beta<1$ and $\alpha<\beta \leq 2 \alpha+1$, then from (2.4) and (2.6) we clearly see that

$$
\begin{aligned}
& h_{2}(0) \leq 0 \\
& h_{2}^{\prime}(t)<0 .
\end{aligned}
$$

Therefore, $h(t)<0$ for $t \in(0, \infty)$, which follows from (2.7) and (2.8) together with (2.1) and (2.2).

Case 2 If $0<\beta<1$ and $\beta \leq \alpha$, then (2.5) and (2.6) lead to

$$
\begin{aligned}
& \lim _{t \rightarrow+\infty} h_{2}(t)=-\beta(\alpha+1)<0, \\
& h_{2}^{\prime}(t) \geq 0 .
\end{aligned}
$$

Therefore, $h(t)<0$ for $t \in(0, \infty)$, which follows from (2.9) and (2.10) together with (2.1) and (2.2).

Case 3 If $1<\beta \leq \alpha$, then (2.4) and (2.6) lead to

$$
\begin{aligned}
& h_{2}(0)<0, \\
& h_{2}^{\prime}(t) \leq 0 .
\end{aligned}
$$

From equations (2.1) and (2.2) together with inequalities (2.11) and (2.12), we clearly see that $h(t)<0$ for $t \in(0, \infty)$.

Case 4 If $\beta>1$ and $\alpha<\beta \leq \alpha+1$, then we clearly see that

$$
\lim _{t \rightarrow+\infty} h(t)=\beta-\alpha-1 \leq 0 .
$$

From (2.3)-(2.6), we know that

$$
\begin{aligned}
& \lim _{t \rightarrow+\infty} h_{1}(t)=+\infty, \\
& h_{2}(0)<0, \\
& \lim _{t \rightarrow+\infty} h_{2}(t)=+\infty, \\
& h_{2}^{\prime}(t)>0 .
\end{aligned}
$$

From (2.15)-(2.17), we clearly see that there exists $t_{1}>0$ such that $h_{2}(t)<0$ for $t \in(0$, $\left.t_{1}\right)$ and $h_{2}(t)>0$ for $t \in\left(t_{1}, \infty\right)$. Hence, $h_{1}(t)$ is strictly decreasing in $\left[0, t_{1}\right]$ and strictly increasing in $\left[t_{1}, \infty\right)$. 
From (2.2) and (2.14) together with the monotonicity of $h_{1}(t)$, we know that there exists $t_{2}>0$ such that $h_{1}(t)<0$ for $t \in\left(0, t_{2}\right)$ and $h_{1}(t)>0$ for $t \in\left(t_{2}, \infty\right)$. Hence, $h(t)$ is strictly decreasing in $\left[0, t_{2}\right]$ and strictly increasing in $\left[t_{2}, \infty\right)$.

Therefore, $h(t)<0$ for $t \in(0, \infty)$ follows from (2.1) and (2.13) together with the monotonicity of $h(t)$.

(2) If $\alpha+1<\beta<2 \alpha+1$, then we clearly see that

$$
\lim _{t \rightarrow+\infty} h(t)=\beta-\alpha-1>0
$$

and (2.14)-(2.17) hold again. From the proof of Case 4 in Lemma 2.1(1), we know that there exists $\lambda>0$ such that $h(t)$ is strictly decreasing in $[0, \lambda]$ and strictly increasing in $[\lambda, \infty)$.

Therefore, Lemma 2.1(2) follows from (2.1) and (2.18) together with the monotonicity of $h(t)$.

(3) If $\beta \geq \alpha+1$ and $\beta \geq 2 \alpha+1$, then we divide the proof into three cases.

Case I If $\beta>1$ and $\beta \geq 2 \alpha+1$, then

$$
\beta>\alpha
$$

and it follows from (2.4) that

$$
h_{2}(0) \geq 0 \text {. }
$$

Equation (2.6) and inequality (2.19) lead to

$$
h_{2}^{\prime}(t)>0 .
$$

Therefore, $h(t)>0$ for $t \in(0, \infty)$ follows from (2.1), (2.2), (2.20), and (2.21).

Case II If $0<\beta<1$ and $\alpha \leq-1$, then from (2.5) and (2.6) we clearly see that

$$
\begin{aligned}
& \lim _{t \rightarrow+\infty} h_{2}(t)=-\beta(\alpha+1) \geq 0, \\
& h_{2}^{\prime}(t)<0 .
\end{aligned}
$$

Inequalities (2.22) and (2.23) imply that

$$
h_{2}(t)>0
$$

for $t \in(0, \infty)$.

Therefore, $h(t)>0$ for $t \in(0, \infty)$ follows from (2.1) and (2.2) together with (2.24).

Case III If $0<\alpha+1 \leq \beta<1$, then we clearly see that

$$
\lim _{t \rightarrow+\infty} h(t)=\beta-\alpha-1 \geq 0 .
$$

It follows from (2.3)-(2.6) that

$$
\begin{aligned}
& \lim _{t \rightarrow+\infty} h_{1}(t)=-\infty, \\
& h_{2}(0)=\beta(\beta-2 \alpha-1)>0, \\
& \lim _{t \rightarrow+\infty} h_{2}(t)=-\beta(\alpha+1)<0, \\
& h_{2}^{\prime}(t)<0 .
\end{aligned}
$$


Inequalities (2.27)-(2.29) imply that there exists $t_{3}>0$ such that $h_{2}(t)>0$ for $t \in\left(0, t_{3}\right)$ and $h_{2}(t)<0$ for $t \in\left(t_{3}, \infty\right)$. Hence, $h_{1}(t)$ is strictly increasing in $\left[0, t_{3}\right]$ and strictly decreasing in $\left[t_{3}, \infty\right)$.

It follows from (2.2) and (2.26) together with the monotonicity of $h_{1}(t)$ that there exists $t_{4}>0$ such that $h_{1}(t)>0$ for $t \in\left(0, t_{4}\right)$ and $h_{1}(t)<0$ for $t \in\left(t_{4}, \infty\right)$. Hence, $h(t)$ is strictly increasing in $\left[0, t_{4}\right]$ and strictly decreasing in $\left[t_{4}, \infty\right)$.

Therefore, $h(t)>0$ for $t \in(0, \infty)$ follows from (2.1) and (2.25) together with the monotonicity of $h(t)$.

(4) If $2 \alpha+1<\beta<\alpha+1$, then we clearly see that

$$
\lim _{t \rightarrow+\infty} h(t)=\beta-\alpha-1<0
$$

and (2.26)-(2.29) hold again.

From the proof of Case III in Lemma 2.1(3) we know that there exists $\mu>0$ such that $h(t)$ is strictly increasing in $[0, \mu]$ and strictly decreasing in $[\mu, \infty)$.

Therefore, Lemma 2.1(4) follows from (2.1) and (2.30) together with the monotonicity of $h(t)$.

\section{Proof of Theorem 1.1}

Proof of Theorem 1.1 Let $E_{1}=\{(\alpha, \beta): 0<\beta<1, \beta \geq \alpha+1\}, E_{2}=\{(\alpha, \beta): \beta>1, \beta \geq 2 \alpha$ $+1\}, E_{3}=\{(\alpha, \beta): \alpha<0, \beta=1\}, E_{4}=\{(\alpha, \beta): \alpha=0, \beta=1\}, E_{5}=\{(\alpha, \beta): \alpha+1<\beta$ $<2 \alpha+1\}, E_{6}=\{(\alpha, \beta): \beta>0,2 \alpha+1<\beta<\alpha+1\}, E_{7}=\{(\alpha, \beta): 0<\beta<1, \beta \leq 2 \alpha+$ $1\}, E_{8}=\{(\alpha, \beta): \beta>1, \beta \leq \alpha+1\}$ and $E_{9}=\{(\alpha, \beta): \alpha>0, \beta=1\}$. Then

$$
\begin{gathered}
\{(\alpha, \beta): \alpha \in \mathbb{R}, \beta>0\}=\cup_{i=1}^{9} E_{i}, \\
\{(\alpha, \beta): \beta>0, \beta \geq 2 \alpha+1, \beta \geq \alpha+1\} \backslash\{(\alpha, \beta): \alpha=0, \beta=1\}=E_{1} \cup E_{2} \cup E_{3}, \\
\{(\alpha, \beta): \beta>0, \beta \leq 2 \alpha+1, \beta \leq \alpha+1\} \backslash\{(\alpha, \beta): \alpha=0, \beta=1\}=E_{7} \cup E_{8} \cup E_{9} .
\end{gathered}
$$

(1) We divide the proof of Theorem 1.1(1) into five cases.

Case 1.1 $(\alpha, \beta) \in E_{1} \cup E_{2}$. From (1.1), (1.2), and applying

$$
\psi^{m}(x)=(-1)^{m+1} \int_{0}^{\infty} \frac{t^{m}}{1-e^{-t}} e^{-x t} d t \quad(x>0, m=1,2, \ldots),
$$

we obtain for $n \geq 1$,

$$
\begin{aligned}
& (-1)^{n}\left[\log F_{\alpha, \beta}(x)\right]^{(n)} \\
= & (-1)^{n}\left[(-1)^{n-1} \frac{\alpha(n-1) !}{x^{n}}+\beta \psi^{(n-1)}(x)-\beta^{n} \psi^{(n-1)}(\beta x)\right] \\
= & -\alpha \int_{0}^{\infty} s^{n-1} e^{-x s} d s+\beta \int_{0}^{\infty} \frac{s^{n-1}}{1-e^{-s}} e^{-x s} d s-\beta^{n} \int_{0}^{\infty} \frac{t^{n-1}}{1-e^{-t}} e^{-\beta x t} d t \\
= & -\alpha \beta^{n} \int_{0}^{\infty} t^{n-1} e^{-\beta x t} d t+\beta^{n+1} \int_{0}^{\infty} \frac{t^{n-1}}{1-e^{-\beta t}} e^{-\beta x t} d t-\beta^{n} \int_{0}^{\infty} \frac{t^{n-1}}{1-e^{-t}} e^{-\beta x t} d t \\
= & \beta^{n} \int_{0}^{\infty} \frac{t^{n-1} e^{-\beta x t}}{\left(1-e^{-t}\right)\left(1-e^{-\beta t}\right)} h(t) d t,
\end{aligned}
$$

where

$$
h(t)=-\alpha e^{-(\beta+1) t}+(\alpha+1) e^{-\beta t}+(\alpha-\beta) e^{-t}-\alpha+\beta-1 .
$$


Therefore, $F_{\alpha, \beta}(x)$ is strictly logarithmically completely monotonic on $(0, \infty)$, which follows from (3.1) and (3.2) together with Lemma 2.1(3).

Case $1.2(\alpha, \beta) \in E_{3}$. Then we clearly see that

$$
(-1)^{n}\left[\log F_{\alpha, \beta}(x)\right]^{(n)}=(-1)^{n} \frac{\alpha(n-1) !(-1)^{n-1}}{x^{n}}=-\frac{\alpha(n-1) !}{x^{n}}>0
$$

for all $x>0$.

Therefore, $F_{\alpha, \beta}(x)$ is strictly logarithmically completely monotonic on $(0, \infty)$, which follows from (3.3).

Case $1.3(\alpha, \beta) \in E_{4}$. Then $F_{\alpha, \beta}(x)=1$ and

$$
(-1)^{n}\left[\log F_{\alpha, \beta}(x)\right]^{(n)}=0 .
$$

Therefore, $F_{\alpha, \beta}(x)$ is not strictly logarithmically completely monotonic on $(0, \infty)$, which follows from (3.4).

Case 1.4 $(\alpha, \beta) \in E_{5} \cup E_{6} \cup E_{7} \cup E_{8}$. Then $F_{\alpha, \beta}(x)$ is not strictly logarithmically completely monotonic on $(0, \infty)$, which follows from Lemmas 2.1(2), 2.1(4), 2.1(1), and equations (3.1) and (3.2).

Case $1.5(\alpha, \beta) \in E_{9}$. Then

$$
(-1)^{n}\left[\log F_{\alpha, \beta}(x)\right]^{(n)}=-\frac{\alpha(n-1) !}{x^{n}}<0
$$

for all $x>0$.

Therefore, $F_{\alpha, \beta}(x)$ is not strictly logarithmically completely monotonic on $(0, \infty)$, which follows from (3.5).

(2) We divide the proof of Theorem 1.1(2) into five cases.

Case 2.1 $(\alpha, \beta) \in E_{7} \cup E_{8}$. Then from (3.1) we get

$$
\begin{aligned}
& (-1)^{n}\left\{\log \left[F_{\alpha, \beta}(x)\right]^{-1}\right\}^{(n)} \\
= & -\beta^{n} \int_{0}^{\infty} \frac{t^{n-1} e^{-\beta x t}}{\left(1-e^{-t}\right)\left(1-e^{-\beta t}\right)} h(t) d t,
\end{aligned}
$$

where

$$
h(t)=-\alpha e^{-(\beta+1) t}+(\alpha+1) e^{-\beta t}+(\alpha-\beta) e^{-t}-\alpha+\beta-1 .
$$

Therefore, $\left[\mathrm{F}_{\alpha, \beta}(x)\right]^{-1}$ is strictly logarithmically completely monotonic on $(0, \infty)$, which follows from (3.6) and (3.7) together with Lemma 2.1(1).

Case 2.2 $(\alpha, \beta) \in E_{9}$. Then

$$
(-1)^{n}\left\{\log \left[F_{\alpha, \beta}(x)\right]^{-1}\right\}^{(n)}=\frac{\alpha(n-1) !}{x^{n}}>0
$$

for all $x>0$.

Therefore, $\left[F_{\alpha, \beta}(x)\right]^{-1}$ is strictly logarithmically completely monotonic on $(0, \infty)$, which follows from (3.8).

Case $2.3(\alpha, \beta) \in E_{4}$. Then $\left[\mathrm{F}_{\alpha, \beta}(x)\right]^{-1}=1$ and

$$
(-1)^{n}\left\{\log \left[F_{\alpha, \beta}(x)\right]^{-1}\right\}^{(n)}=0 .
$$


Therefore, $\left[F_{\alpha, \beta}(x)\right]^{-1}$ is not strictly logarithmically completely monotonic on $(0, \infty)$, which follows from (3.9).

Case 2.4 $(\alpha, \beta) \in E_{1} \cup E_{2} \cup E_{5} \cup E_{6}$. Then $\left[F_{\alpha, \beta}(x)\right]^{-1}$ is not strictly logarithmically completely monotonic on $(0, \infty)$, which follows from equations (3.6) and (3.7) and Lemmas 2.1(3), 2.1(2) and 2.1(4).

Case $2.5(\alpha, \beta) \in E_{3}$. Then

$$
(-1)^{n}\left\{\log \left[F_{\alpha, \beta}(x)\right]^{-1}\right\}^{(n)}=\frac{\alpha(n-1) !}{x^{n}}<0
$$

for all $x>0$.

Therefore, $\left[F_{\alpha, \beta}(x)\right]^{-1}$ is not strictly logarithmically completely monotonic on $(0, \infty)$, which follows from (3.10).

\section{Acknowledgements}

This study is partly supported by the Natural Science Foundation of China (Grant no. 11071069), the Innovation Team Foundation of the Department of Education of Zhejiang Province (Grant no. T200924), and the Natural Science Foundation of the Department of Education of Zhejiang Province (Grant no. Y200805602).

\section{Authors' contributions}

Y-PL carried out the proof of Theorem 1.1 in this paper. T-CS carried out the proof of Lemma 2.1 in this paper. Y-MC provided the main idea of this paper. All authors read and approved the final manuscript.

\section{Competing interests}

The authors declare that they have no competing interests.

Received: 9 February 2011 Accepted: 22 August 2011 Published: 22 August 2011

\section{References}

1. Whittaker, ET, Watson, GN: A Course of Modern Analysis. Cambridge University Press, New York (1962)

2. Chu, Y-M, Zhang, X-M, Zhang, Zh-H: The geometric convexity of a function involving gamma function with applications. Commun Korean Math Soc. 25(3), 373-383 (2010). doi:10.4134/CKMS.2010.25.3.373

3. Zhang, X-M, Chu, Y-M: A double inequality for gamma function. J Inequal Appl 2009, 1-7 (2009). Article ID 503782

4. Zhao, T-H, Chu, Y-M, Jiang, Y-P: Monotonic and logarithmically convex properties of a function involving gamma functions. J Inequal Appl 2009, 1-13 (2009). Article ID 728612

5. Zhang, X-M, Chu, Y-M: An inequality involving the gamma function and the psi function. Int J Mod Math. 3(1), 67-73 (2008)

6. Chu, Y-M, Zhang, X-M, Tang, X-M: An elementary inequality for psi function. Bull Inst Math Acad Sin. 3(3), 373-380 (2008)

7. Song, Y-Q, Chu, Y-M, Wu, L-L: An elementary double inequality for gamma function. Int J Pure Math. 38(4), 549-554 (2007)

8. Guo, B-N, Qi, F: Two new proofs of the complete monotonicity of a function involving the PSI function. Bull Korean Math Soc. 47(1), 103-111 (2010). doi:10.4134/BKMS.2010.47.1.103

9. Chen, Ch-P, Qi, F, Srivastava, HM: Some properties of functions related to the gamma and psi functions. Int Trans Spec Funct. 21(1-2), 153-164 (2010)

10. Qi, F: A completely monotonic function involving the divided difference of the psi function and an equivalent inequality involving sums. ANZIAM J. 48(4), 523-532 (2007). doi:10.1017/S1446181100003199

11. Qi, F, Guo, B-N: Monotonicity and convexity of ratio between gamma functions to different powers. J Indones Math Soc. 11(1), 39-49 (2005)

12. Chen, Ch-P, Qi, F: Inequalities relating to the gamma function. Aust J Math Anal Appl 1(1), 1-7 (2004). Article 3

13. Guo, B-N, Qi, F: Inequalities and monotonicity for the ratio of gamma functions. Taiwan J Math. 7(2), 239-247 (2003)

14. Qi, F: Monotonicity results and inequalities for the gamma and incomplete gamma functions. Math Inequal Appl. 5(1), 61-67 (2002)

15. Qi, F, Mei, J-Q: Some inequalities of the incomplete gamma and related functions. Z Anal Anwendungen. 18(3), 793-799 (1999)

16. Qi, F, Guo, S-L: Inequalities for the incomplete gamma and related functions. Math Inequal Appl. 2(1), 47-53 (1999)

17. Alzer, H: Some gamma function inequalities. Math Comp. 60(201), 337-346 (1993). doi:10.1090/S0025-5718-19931149288-7

18. Alzer, H: On some inequalities for the gamma and psi functions. Math Comp. 66(217), 373-389 (1997). doi:10.1090/ S0025-5718-97-00807-7

19. Anderson, GD, Qiu, S-L: A monotonicity property of the gamma function. Proc Am Math Soc. 125(11), 3355-3362 (1997). doi:10.1090/S0002-9939-97-04152-X

20. Kershaw, D: Some extensions of W. Gautschi's inequalities for the gamma function. Math Comp. 41(164), 607-611 (1983) 
21. Merkle, M: Logarithmic convexity and inequalities for the gamma function. J Math Anal Appl. 203(2), 369-380 (1996). doi:10.1006/jmaa.1996.0385

22. Palumbo, B: A generalization of some inequalities for the gamma function. J Comput Appl Math. 88(2), 255-268 (1998). doi:10.1016/50377-0427(97)00187-8

23. Alzer, H, Berg, Ch: Some classes of completely monotonic functions II. Ramanujan J. 11(2), 225-248 (2006). doi:10.1007/ s11139-006-6510-5

24. Alzer, H: Sharp inequalities for the digamma and polygamma functions. Forum Math. 16(2), 181-221 (2004). doi:10.1515/form.2004.009

25. Alzer, H, Batir, N: Monotonicity properties of the gamma function. Appl Math Lett. 20(7), 778-781 (2007). doi:10.1016/j. aml.2006.08.026

26. Clark, WE, Ismail, MEH: Inequalities involving gamma and psi functions. Anal Appl. 1(1), 129-140 (2003). doi:10.1142/ S0219530503000041

27. Elbert, Á, Laforgia, A: On some properties of the gamma function. Proc Am Math Soc. 128(9), 2267-2673 (2000)

28. Bustoz, J, Ismail, MEH: On gamma function inequalities. Math Comp. 47(176), 659-667 (1986). doi:10.1090/50025-57181986-0856710-6

29. Ismail, MEH, Lorch, L, Muldoon, ME: Completely monotonic functions associated with the gamma function and its qanalogues. J Math Anal Appl. 116(1), 1-9 (1986). doi:10.1016/0022-247X(86)90042-9

30. Babenko, VF, Skorokhodov, DS: On Kolmogorov-type inequalities for functions defined on a semiaxis. Ukr Math J. 59(10), 1299-1312 (2007)

31. Muldoon, ME: Some monotonicity properties and characterizations of the gamma function. Aequationes Math. 18(1-2), 54-63 (1978). doi:10.1007/BF01844067

32. Qi, F, Yang, Q, Li, W: Two logarithmically completely monotonic functions connected with gamma function. Int Trans Spec Funct. 17(7), 539-542 (2006). doi:10.1080/10652460500422379

33. Qi, F, Niu, D-W, Cao, J: Logarithmically completely monotonic functions involving gamma and polygamma functions. J Math Anal Approx Theory. 1(1), 66-74 (2006)

34. Qi, F, Chen, Sh-X, Cheung, W-S: Logarithmically completely monotonic functions concerning gamma and digamma functions. Int Trans Spec Funct. 18(6), 435-443 (2007). doi:10.1080/10652460701318418

35. Qi, F: A class of logarithmically completely monotonic functions and the best bounds in the first Kershaw's double inequality. J Comput Appl Math. 206(2), 1007-1014 (2007). doi:10.1016/j.cam.2006.09.005

36. Chen, Ch-P, Qi, F: Logarithmically complete monotonicity properties for the gamma function. Aust J Math Anal Appl 2(2), 1-9 (2005). Article 8

37. Chen, Ch-P, Qi, F: Logarithmically completely monotonic functions relating to the gamma function. J Math Anal Appl. 321(1), 405-411 (2006). doi:10.1016/j.jmaa.2005.08.056

38. Merkle, M: On log-convexity of a ratio of gamma functions. Univ Beograd Publ Elektrotehn Fak Ser Mat. 8, 114-119 (1997)

39. Chen, Ch-P: Complete monotonicity properties for a ratio of gamma functions. Univ Beograd Publ Elektrotehn Fak Ser Mat. 16, 26-28 (2005)

40. Li, A-J, Chen, Ch-P: Some completely monotonic functions involving the gamma and polygamma functions. J Korean Math Soc. 45(1), 273-287 (2008). doi:10.4134/JKMS.2008.45.1.273

doi:10.1186/1029-242X-2011-36

Cite this article as: Lv et al: Necessary and sufficient conditions for a class of functions and their reciprocals to be logarithmically completely monotonic. Journal of Inequalities and Applications 2011 2011:36.

\section{Submit your manuscript to a SpringerOpen ${ }^{\circ}$ journal and benefit from:}

- Convenient online submission

- Rigorous peer review

- Immediate publication on acceptance

- Open access: articles freely available online

- High visibility within the field

- Retaining the copyright to your article

Submit your next manuscript at $\gg$ springeropen.com 